\title{
Numerical study of MHD flow and heat transfer through porous medium between two parallel plates with Hall and ion slip effects
}

\author{
Odelu Ojjela \\ Department of Applied Mathematics \\ Defence Institute of Advanced Technology \\ (Deemed University), Pune-411025, India \\ odelu3@yahoo.co.in
}

\author{
N Naresh Kumar \\ Department of Applied Mathematics \\ Defence Institute of Advanced Technology \\ (Deemed University), Pune-411025, India \\ nareshkumarn622@gmail.com
}

\begin{abstract}
This paper studies the effects of Hall and ion slip on two dimensional incompressible flow and heat transfer of an electrically conducting viscous fluid in a porous medium between two parallel plates, generated due to periodic suction and injection at the plates. The flow field, temperature and pressure are assumed to be periodic functions in $e^{i \omega t}$ and the plates are kept at different but constant temperatures. A numerical solution for the governing nonlinear ordinary differential equations is obtained using quasilinearization method. The graphs for velocity, temperature distribution and skin friction are presented for different values of the fluid and geometric parameters.
\end{abstract}

Keywords- Unsteady; Porous medium; Temperature distribution; Skin friction; Hall effect; ion slip effect

\section{INTRODUCTION}

The flow and temperature distribution through porous channels is of great importance in technological and biological flows, for example motion of water waves over a shallow beach, the mechanics of cochlea in the human ear, transpiration cooling, filtration, food preservation, petroleum industry, cosmetic industry, polymer technology, the flow of blood in the arteries and artificial dialysis, magnetohydrodynamic (MHD) generators, etc,. The problem of two dimensional incompressible laminar flow between parallel plates with either suction or injection was considered by many researchers. Berman [1] investigated the flow of two dimensional steady incompressible laminar viscous fluid through a porous channel where both channel plates have equal permeability and the flow at the center line of the channel attains maximum. Later Yuan [2] extended the problem for different values of suction and injection Reynolds numbers. Terril and Shresta [3] considered the incompressible viscous fluid through porous walls when suction normal velocities are distinct. Gersten and Gross [4] have examined a three dimensional incompressible laminar viscous fluid flow and temperature distribution with transverse sinusoidal suction velocity. Alpher [5] examined an incompressible laminar flow and convection heat transfer between parallel plates through a transverse magnetic field. Cox [6] discussed a two dimensional incompressible viscous fluid between two parallel porous walls with symmetric and asymmetric suction. Singh and Sharma [7] solved numerically the steady incompressible laminar flow of a non-Newtonian fluid and temperature distribution in a porous annulus. Ramanamurthy et al. [8] studied the two dimensional incompressible viscous fluid between porous parallel plates due to periodic suction and injection at the plates, the problem is analyzed up to second order. Vidhya and Kesavan [9] considered an incompressible viscous fluid flow and temperature distribution in a porous medium between two vertical parallel plates and the problem is analyzed analytically. Attia and Kotb[10] considered incompressible steady and unsteady MHD fluid flow and temperature distribution between porous parallel plates with suction or injection. Attia [11] considered an unsteady transient MHD flow and heat transfer between parallel plates with Hall and ion slip effects and obtained the analytical and numerical solutions for velocity and temperature distribution respectively. Bhat and Mittal [12] analyzed the effects of an electrically conducting convective heat transfer in a uniform wall with Hall and ion slip. Jha and Apere [13] examined the effects of Hall and ion slip on unsteady MHD Couette flow of rotating fluid and obtained an analytical solution by Laplace transform. Ram [14] studied the effects of Hall and ion slip on steady incompressible electrically conducting rotating fluid with temperature distribution and obtained an analytical solution. Ghara et. al [15] investigated an unsteady electrically conducting Couette flow between porous parallel plates with Hall and ion slip effects and the solution of flow field equations obtained by Laplace transform technique.

In this paper we consider the unsteady two dimensional flow and temperature distribution of an electrically conducting viscous fluid between two parallel horizontal plates with Hall and ion slip currents. The solution is obtained numerically using quasilinearization technique and the effects of suction Reynolds number, suction and injection ratio, Hartman number, inverse Darcy parameter, frequency parameter, Hall and ion slip parameters on velocity, 
temperature distribution and skin friction are studied and shown graphically.

\section{Nomenclature}

h

$V_{2}$

$V_{1}$

$D^{-1}$

$\bar{Q}$

C

E

$\mathrm{k}$

$\mathrm{k}_{1}$

$\mathrm{t}$

$\mathrm{u}$

$\mathrm{V}$

$U_{0}$

$\operatorname{Pr}$

$\mathrm{R}$

$\bar{B}$

b

$B_{0}$

$\bar{E}$

$\mathrm{Ha}$
Distance between parallel plates

Suction velocity

Injection velocity

Inverse Darcy parameter, $\frac{h^{2}}{k_{1}}$

Suction and injection ratio, $1-\frac{V_{1}}{V_{2}}$

Fluid pressure

Velocity vector

Specific heat at constant temperature

Eckert number, $\frac{\mu V_{2}}{\rho h c\left(\theta_{2}-\theta_{1}\right)}$

Thermal conductivity

Permeability parameter

Time

Velocity component in $\mathrm{x}$ - direction

Velocity component in y-direction

Entrance velocity

Prandtl number, $\frac{\mu C}{k}$

Suction Reynolds number, $\frac{\rho V_{2} h}{\mu}$

Current density

Total magnetic field.

Induced magnetic field

Magnetic flux density

Electric field

Hartmann number, $B_{0} h \sqrt{\frac{\sigma}{\mu}}$.

\section{Greek Letters}

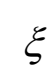

$\tau$

$\mu$

$\omega$

$\sigma$

$\alpha$

$\theta$

$\theta_{1}$

$\theta_{2}$

$\theta^{*}$

$\beta_{\mathrm{i}}$

$\beta_{\mathrm{e}}$

$\alpha_{\mathrm{e}}$
Dimensionless y coordinate, $\frac{y}{h}$

Force stress tensor

Dimensionless axial variable, $\left(\frac{U_{0}}{a V_{2}}-\frac{x}{h}\right)$

Fluid density

Fluid viscosity

Frequency

Electric conductivity

Non dimensional frequency parameter, $\omega t$

Temperature

Temperature at the lower plate

Temperature at the upper plate

Dimensionless temperature, $\frac{\theta-\theta_{1}}{\theta_{2}-\theta_{1}}$

Ion slip parameter

Hall parameter

Hall and ion-slip parameter, $1+\beta_{\mathrm{i}} \beta_{\mathrm{e}}$

\section{FORMULATION OF THE PROBLEM}

The unsteady laminar incompressible electrically conducting viscous fluid is assumed to be flowing between two parallel porous plates located at the $\mathrm{y}=0$ and $\mathrm{y}=\mathrm{h}$ as shown in the fig.1.The two plates are kept at two different constant temperatures $\theta_{1}$ for the lower plate and $\theta_{2}$ for the upper plate. Let there be a periodic suction at the upper plate and periodic injection at the lower plate with velocities Real $\left(\mathrm{V}_{2} e^{i \omega t}\right)$ and Real $\left(\mathrm{V}_{1} e^{i \omega t}\right)$ respectively.

The governing equations of an incompressible electrically conducting viscous fluid flow and energy in a porous medium are

$$
\begin{gathered}
\nabla \cdot \bar{Q}=0 \\
\rho\left[\frac{\partial \bar{Q}}{\partial t}+(\bar{Q} \cdot \nabla) \bar{Q}\right]=-\nabla \mathrm{p}-\mu \nabla \times \nabla \times \bar{Q}
\end{gathered}
$$




$$
\begin{gathered}
-\frac{\mu}{k_{1}} \bar{Q}+J \times \bar{B} \\
\rho c\left[\frac{\partial \theta}{\partial t}+(\bar{Q} \cdot \nabla) \theta\right]=\mu\left[(\nabla \bar{Q}):(\nabla \bar{Q})^{\mathrm{T}}+(\nabla \bar{Q}):(\nabla \bar{Q})\right]+ \\
k \nabla^{2} \theta+\frac{\mu}{k_{1}} \bar{Q}^{2}+\frac{1}{\sigma} J^{2}
\end{gathered}
$$

where $\bar{Q}$ is the velocity vector,$\rho$ is the density, $\mathrm{p}$ is the fluid pressure, $\mathrm{k}$ is the thermal conductivity, $\mu$ is the fluid viscosity, $c$ is the specific at constant temperature, $\theta$ is the temperature, $\mathrm{k}_{1}$ is the permeability parameter, $\mathrm{J}$ is current density, $\bar{B}$ is total magnetic field and $\sigma$ is the electric conductivity.

Since the flow is along $\mathrm{x}$ - direction, the flow variables are assumed to be independent of the coordinate $\mathrm{z}$. We choose the velocity vector as $\bar{Q}=u(x, y . t) \hat{i}+v(x, y, t) \hat{j}$ and temperature function as $\theta(x, y, t)$ and neglecting the displacement current, the Maxwell equations and the generalized Ohm's law are

$$
\begin{gathered}
\nabla \cdot \bar{B}=0, \nabla \times \bar{B}=\mu_{m} \bar{J}, \nabla \times \bar{E}=\frac{\partial \bar{B}}{\partial t}, \\
\bar{J}=\sigma(\bar{E}+\bar{Q} \times \bar{B})+\frac{\beta_{e}}{B_{0}}(\bar{J} \times \bar{B})-\frac{\beta_{e} \beta_{i}}{B_{0}^{2}}(\bar{J} \times \bar{B}) \times \bar{B}
\end{gathered}
$$

Where $\mu_{m}$ is the magnetic permeability, $\bar{E}$ is the electric field, and $\bar{B}=B_{0}+b$, b is induced magnetic field, $B_{0}$ is magnetic flux density, $\beta_{e}$ is the Hall parameter, $\beta_{i}$ is the ion slip parameter.

Under the assumptions that the induced magnetic field is negligible compared to the applied magnetic field so that magnetic Reynolds number is small, the electric field is zero and magnetic permeability is constant throughout the flow field.

We take the velocity components in $\mathrm{x}$ and $\mathrm{y}$ directions as (1965)

$$
\begin{aligned}
& u=\left(\frac{U_{0}}{a}-\frac{V_{2} x}{h}\right) f^{1}(\xi) e^{i \omega t}, v=V_{2} f(\xi) e^{i \omega t}, \\
& p=p(x, \xi) e^{i \omega t} \text { and } \\
& \theta=\theta_{1}+\frac{\mu V_{2}}{\rho h c}\left[g_{1}(\xi)+\left(\frac{U_{0}}{a V_{2}}-\frac{x}{h}\right)^{2} g_{2}(\xi)\right] e^{i \omega t}(5)
\end{aligned}
$$

The boundary conditions on the velocity and temperature distribution are

$$
\begin{aligned}
& u(x, \xi, t)=0, v(x, \xi, t)=V_{1}, \theta(x, \xi, t)=\theta_{1} \text { at } \xi=0 \\
& u(x, \xi, t)=0, v(x, \xi, t)=V_{2}, \theta(x, \xi, t)=\theta_{2} \text { at } \xi=1(6)
\end{aligned}
$$

Where $\xi=\frac{y}{h}$.

From equations (2) \& (3), we get the following non dimensional equations

$\operatorname{Re}\left[f f^{111}-f^{1} f^{11}\right] \cos \alpha-f^{1 V}+D^{-1} f^{11}+\frac{H a^{2} \alpha_{e}}{\alpha_{e}^{2}+\beta_{e}^{2}} f^{11}=0$

$$
g_{1}^{11}+\operatorname{Re} \operatorname{Pr}\left(4 f^{1^{2}}-f g_{1}^{1}+D^{-1} f^{2}+\frac{H a^{2}}{\alpha_{e}^{2}+\beta_{e}^{2}} f^{2}\right) \cos \alpha=0
$$

$$
g_{2}^{11}+\operatorname{Re} \operatorname{Pr}\left(f^{11^{2}}+2 f^{1} g_{2}-f g_{2}^{1}+D^{-1} f^{2}+\frac{H a^{2}}{\alpha_{e}^{2}+\beta_{e}^{2}} f^{2}\right) \cos \alpha=0
$$

Where prime denotes differentiation with respect to $\xi$, $\operatorname{Re}=\frac{\rho h V_{2}}{\mu}$ is Reynolds number, $\operatorname{Pr}=\frac{\mu c}{k}$ is the Prandtl number, $D^{-1}=\frac{h^{2}}{k_{1}}$ is inverse Darcy's parameter, $\alpha_{e}=1+\beta_{e} \beta_{i}$ is Hall and ion slip parameter and $H a=B_{0} h \sqrt{\frac{\sigma}{\mu}}$ is Hartmann number.

The dimensionless form of temperature from equation (5) can be written as

$$
\theta^{*}=\frac{\theta-\theta_{1}}{\theta_{2}-\theta_{1}}=E\left(g_{1}+\gamma^{2} g_{2}\right)
$$

Where $\mathrm{E}$ is the Eckert number, $\mathrm{E}=\frac{\mu V_{2}}{\rho h c\left(\theta_{2}-\theta_{1}\right)}$

The boundary conditions from eq. (9) in terms of $f, g_{1}$ and $g_{2}$ are

$$
\begin{aligned}
& f(0)=1-a, f(1)=0, f^{1}(0)=0, f^{1}(1)=0, \\
& g_{1}(0)=0, g_{1}(1)=w, g_{2}(0)=0, g_{2}(1)=0
\end{aligned}
$$




\section{SOLUTION OF THE PROBLEM}

The following system of first order linear differential equations are obtained from the non linear equations (7), (8), and (8) by the substitution

$$
\begin{aligned}
& \left(f, f^{1}, f^{11}, f^{111}, f^{1 v}, g_{1}, g_{1}^{1}, g_{2}, g_{2}^{1}\right)= \\
& \left(x_{1}, x_{2}, x_{3}, x_{4}, x_{5}, x_{6}, x_{7}, x_{8}\right) \\
& \frac{d x_{1}}{d \xi}=x_{2}, \frac{d x_{2}}{d \xi}=x_{3}, \frac{d x_{3}}{d \xi}=x_{4}, \\
& \frac{d x_{4}}{d \xi}=D^{-1} x_{3}+\frac{H a^{2} \alpha_{e}}{\alpha_{e}^{2}+\beta_{e}^{2}} x_{3}+\operatorname{Re}\left(x_{1} x_{4}-x_{2} x_{3}\right) \cos \alpha \\
& \frac{d x_{5}}{d \xi}=x_{6},
\end{aligned}
$$

$$
\begin{aligned}
\frac{d x_{6}}{d \xi}= & -2 x_{7}+\operatorname{Re} \operatorname{Pr}\left(-D^{-1} x_{1}^{2}-\frac{H a^{2}}{\alpha_{e}^{2}+\beta_{e}^{2}} x_{1}^{2}\right. \\
& \left.-4 x_{2}^{2}+x_{1} x_{6}\right) \cos \alpha, \frac{d x_{7}}{d \xi}=x_{8},
\end{aligned}
$$

$$
\begin{array}{r}
\frac{d x_{8}}{d \xi}=\operatorname{Re} \operatorname{Pr}\left(-D^{-1} x_{2}^{2}-x_{3}^{2}-2 x_{2} x_{7}+\right. \\
\left.x_{1} x_{8}-\frac{H a^{2}}{\alpha_{e}^{2}+\beta_{e}^{2}} x_{2}^{2}\right) \cos \alpha
\end{array}
$$

the boundary conditions in terms of

$$
\begin{aligned}
& x_{1}, x_{2}, x_{3}, x_{4}, x_{5}, x_{6}, x_{7}, x_{8} \text { are } \\
& x_{1}(0)=1-a, x_{2}(0)=0, x_{5}(0)=0, x_{7}(0)=0 \\
& x_{1}(1)=1, x_{2}(1)=0, x_{5}(0)=w, x_{7}(1)=0
\end{aligned}
$$

Using quasilinearization technique (1965) the system of equations (11) is solved numerically subject to the boundary conditions (12).

\section{RESULTS AND DISCUSSIONS}

To understand the flow characteristics in better way, the numerical results for the axial velocity $\mathrm{u}$, radial velocity $\mathrm{v}$, temperature distribution $\theta$ and skin friction coefficient $\mathrm{S}_{\mathrm{f}}$ are calculated correct to six places of decimal for different values of Hartmann number $\mathrm{Ha}$, suction Reynolds number Re, suction and injection ratio a, frequency parameter $\alpha$, Inverse Darcy parameter $D^{-1}$ and Prandtl number Pr in the domain $[0,1]$.

It is examined that from Fig. 1(a) and 1(b), when the values of $\mathrm{Re}$ increases the velocity decreased and the temperature distribution is increased from lower plate to upper plate. It is observed that from Fig.2(a), 2(b) and 8, when the values of ' $a$ ' increases the velocity is decreased where as the temperature distribution is increased towards the upper plate and the skin friction at lower plate is increased with respect to Re. It is analyzed that from Fig. 3(a), 3(b) and 9 when the values of $\alpha$ increases the velocity and temperature distribution are decreased from lower plate to upper plate and the skin friction at the lower plate is also decreased with respect to Re. It is noted that form Fig. 4(a), 4(b) and 10 when the values of Ha increases the velocity is increased gradually up to the centre of the plates then decreased symmetrically and where as the temperature distribution is decreased from the lower plate to upper plate and the skin friction at the lower plate is increased with respect Re. It examined that from Fig. 5(a), 5(b) and 12 when the values of $\beta_{e}$ increases the velocity is increased where as the temperature is decreased from lower plate to upper plate and the skin friction at the lower plate is also decreased with respect to Re. It examined that from Fig. 6(a), 6(b) and 11 when the values of $\alpha_{e}$ increases the velocity is increased where as the temperature is decreased towards the upper plate and the skin friction at the lower plate is also decreased with respect to Re. It is noticed that From Fig.7(a) ,7(b) and 13 when the values of $D^{-1}$ increases the velocity is decreased where as the temperature distribution is increased from the lower plate to upper plate and the skin friction at the lower plate is increased with respect to Re. It is observed that from Fig.14 when the values of Pr increases the temperature distribution is increased towards the upper plate. 


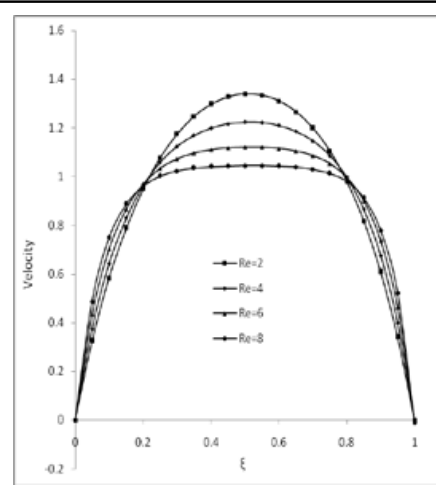

Fig.1(a) Effect of Re on Velocity

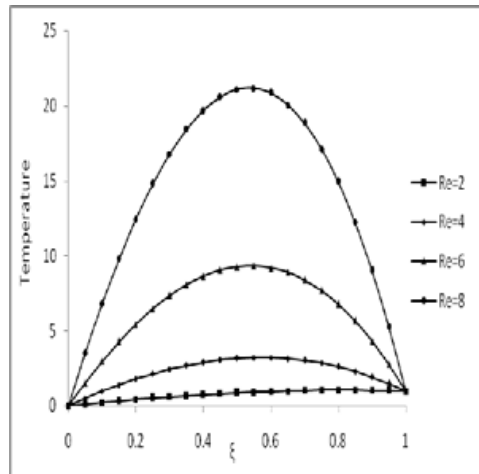

Fig.1(b) Effect of Re on Temperature

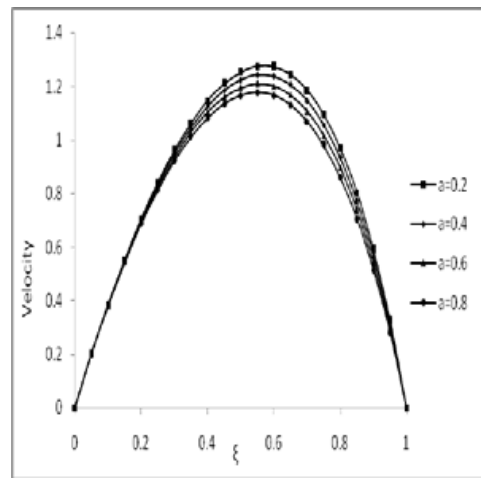

Fig.2(a) Effect of 'a' on Velocity

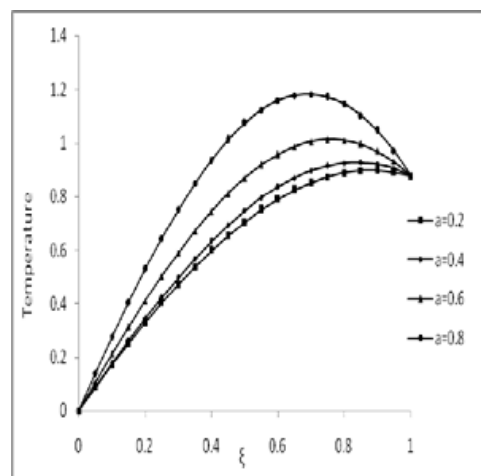

Fig.2(b) Effect of 'a' on Temperature

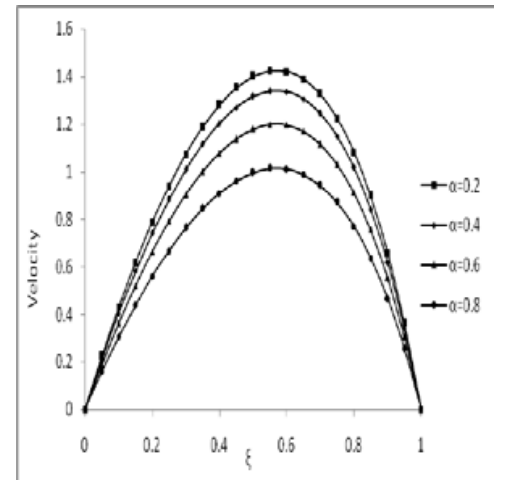

Fig.3(a) Effect of $\alpha$ on Velocity

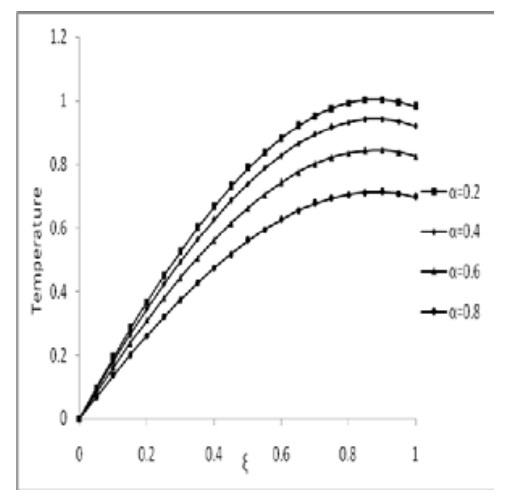

Fig.3(b) Effect of $\alpha$ on Temperature

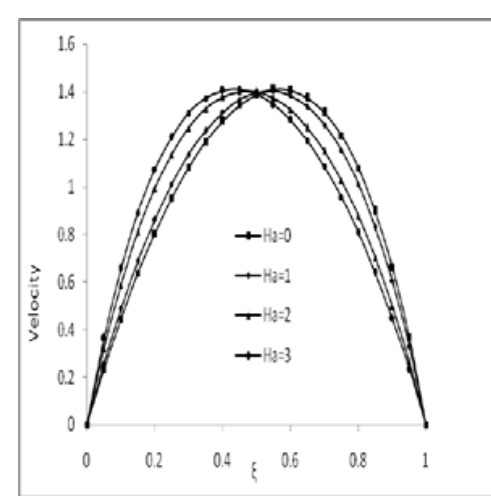

Fig.4(a) Effect of Ha on Velocity

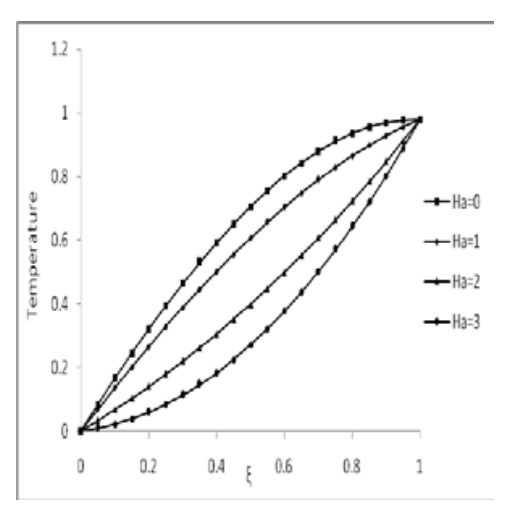

Fig.4(b) Effect of Ha on Temperature 


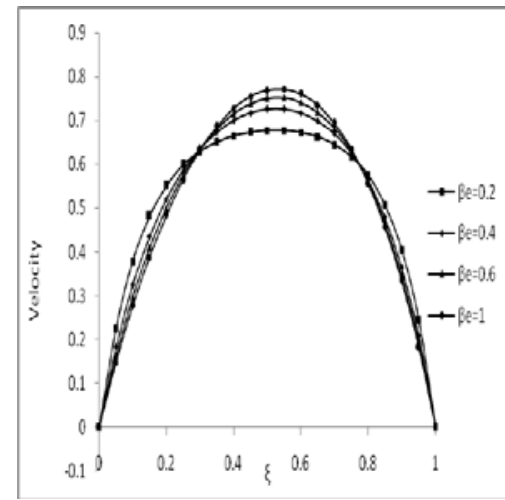

Fig.5(a) Effect of $\beta_{\mathrm{e}}$ on Velocity

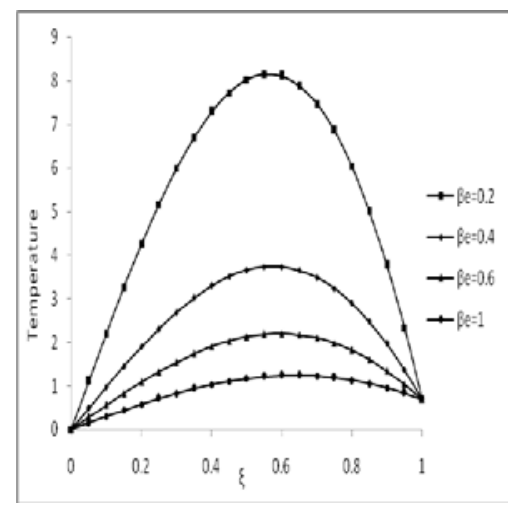

Fig.5(b) Effect of $\beta_{\mathrm{e}}$ on Temperature

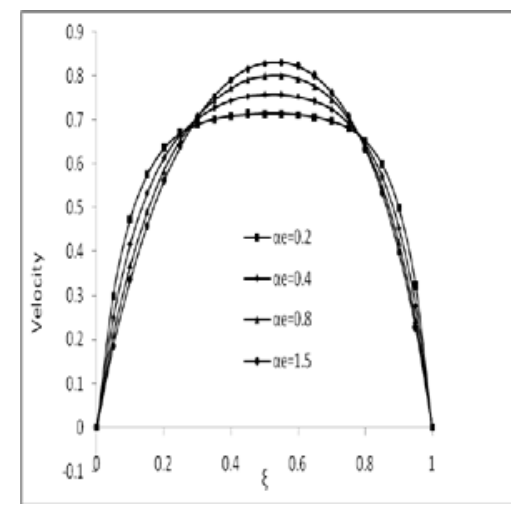

Fig.6(a) Effect of $\alpha_{e}$ on Velocity

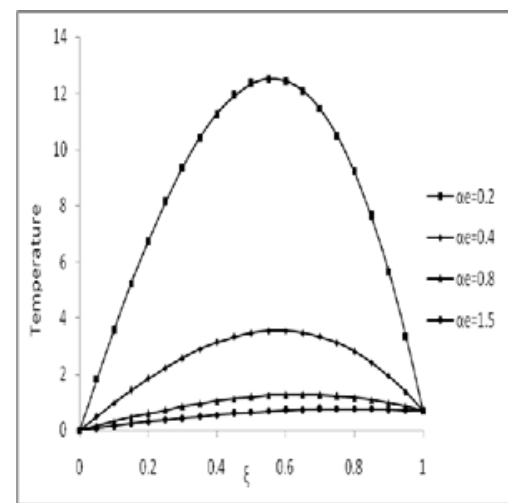

Fig.6(b) Effect of $\alpha_{\mathrm{e}}$ onTemperature

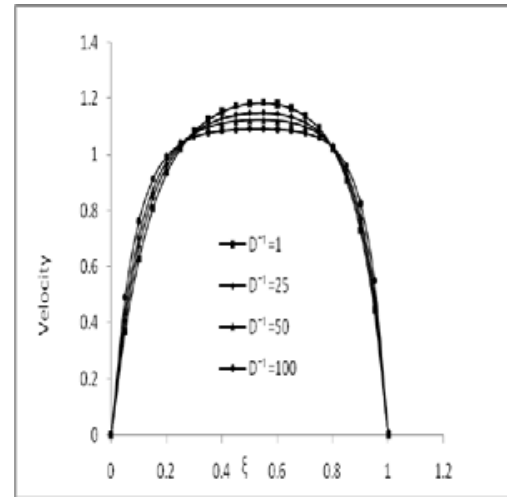

Fig.7(a) Effect of $\mathrm{D}^{-1}$ on Velocity

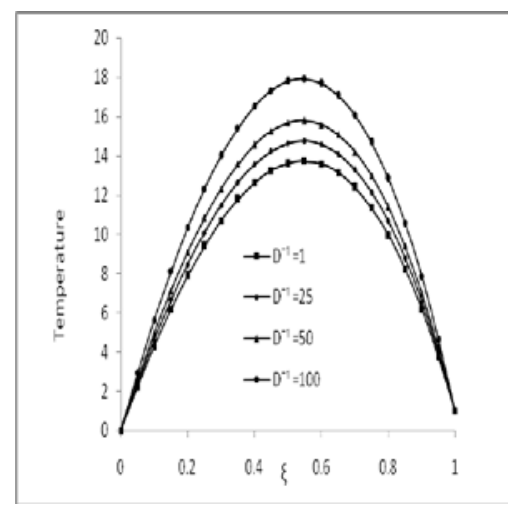

Fig.7(b) Effect of $D^{-1}$ on Temperature

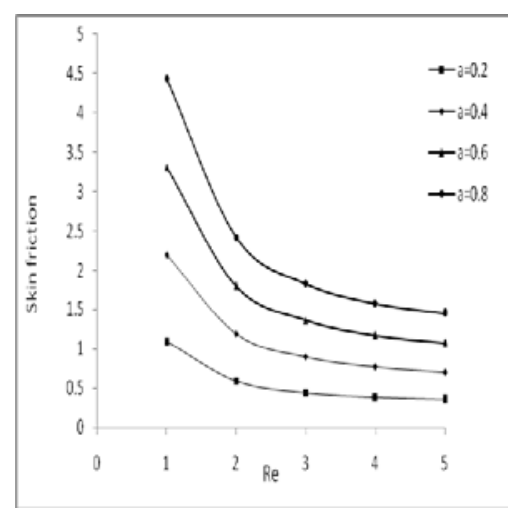

Fig.8 Effect of 'a' on Skin friction

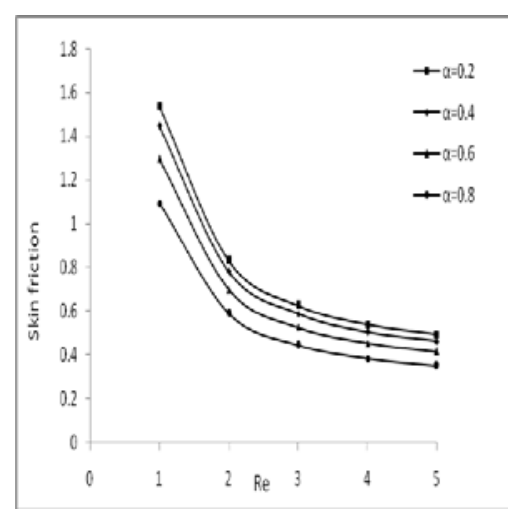

Fig.9 Effect of $\alpha$ on Skin friction 


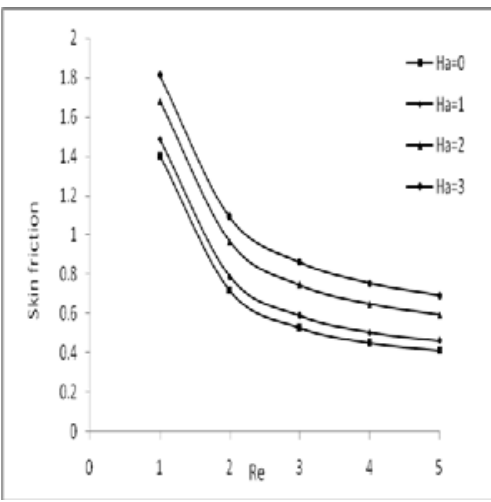

Fig.10 Effect of Ha on Skin friction

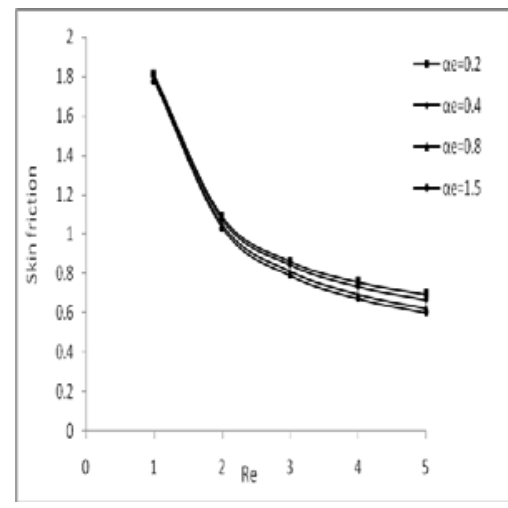

Fig.11 Effect of $\alpha_{\mathrm{e}}$ on Skin friction

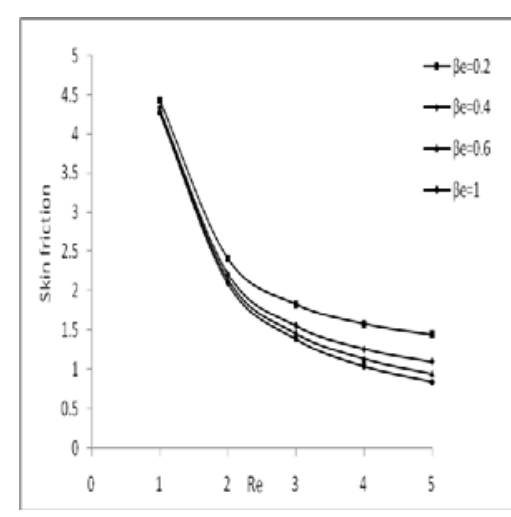

Fig.12 Effect of $\beta_{\mathrm{e}}$ on Skin friction

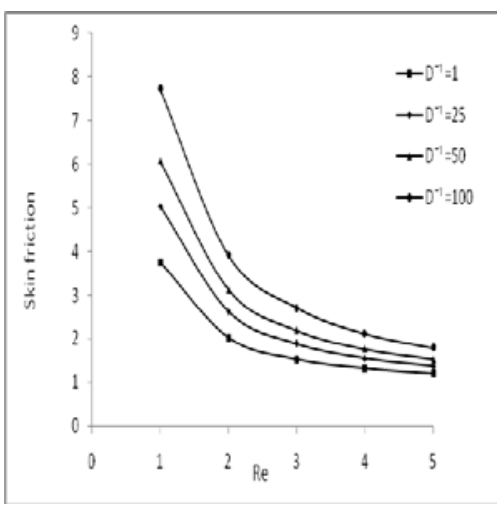

Fig. 13 Effect of $\mathrm{D}^{-1}$ on Skin friction

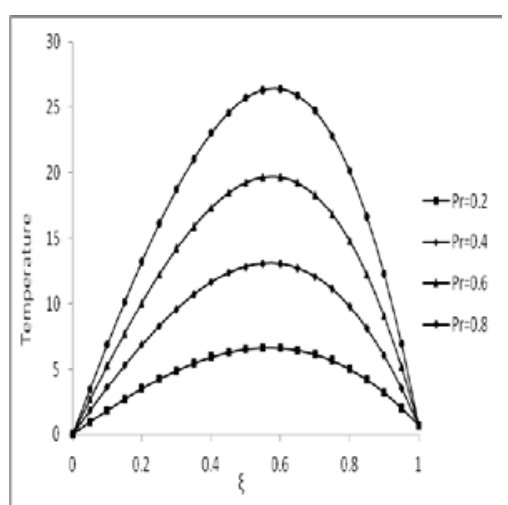

Fig. 14 Effect of Pr on Temperature

REFERENCES

[1] A. S. Berman, Laminar flow in channels with porous walls, Journal of Applied physics, 1953, Vol 24, pp. 1232 - 1235.

[2] S.W.Yuan, Further investigations of laminar flow in channels with porous walls, Journal of Applied physics, 1956, Vol. 27, pp. 267-269.

[3] R.M. Terril and G.M. Shrestha, "Laminar flow through parallel and uniformly porous walls of different permeability”, ZAMP, 1965, Vol.16, pp. $470-482$.

[4] K. Gersten and J.F. Gross,” Flow and heat transfer along a plane wall with periodic suction”, ZAMP, 1974,Vol. 25, pp. 399-408.

[5] R. A. Alpher, "Heat transfer in magnetohydrodynamic flow between parallel plates”, Int. J. Heat Mass transfer, 1961, Vol 3. pp. 108-112

[6] S. M. Cox, "Two dimensional flow of a viscous fluid in a channel with porous walls”, Journal of Fluid Mechanics, 1991, Vol. 227 , pp.1-33.

[7] H. G. Sharma and K. R. Singh, " Heat transfer in the laminar flow of a non-Newtonian fluid in porous annulus by the method of quasilinearization International Journal of Heat and Mass Transfer, 1987, Vol. 30(6) , pp. 1227-1231.

[8] J.V.Ramana Murthy ,N. Srinivasacharyulu and Odelu Ojjela,”Viscous fluid flow between two parallel plates with periodic suction and injection”, AMSE Journal Advances in Modelling Series B, 2007, Vol. 50 No.2, pp. 29-37.

[9] M. Vidhya and Sundarammal Kesavan,"Laminar convection through porous medium between two vertical parallel plates with heat source", 2010, IEEE, pp. 197-200.

[10] H.A. Attia and, N.A. Kotb, "MHD flow between two parallel plates with heat transfer”, Acta Mechanica, 1996, Vol. 117 No.1-4, pp. 215-220.

[11] Hazem A. Attia,'Transient Hartmann Flow with Heat Transfer Considering the Ion Slip”, Physica Scripta., 2002, Vol. 66, 470-475.

[12] A. N. Bhat and M. L. Mittal, "Heat transfer in a mhd channel with Uniform wall heat flux - effects of Hall And ion slip currents”, Int. J. Heat Mass Transfer, 1980, Vol. 23, pp 919-926.

[13] B. K. Jha, C. A. Apere, “Time-dependent MHD Couette flow of rotating fluid with Hall and ion-slip currents”, Appl. Math. Mech. -Engl. Ed., 2012, Vol 33(4), pp. 399-410.

[14] P. C. Ram, "MHD convective flow in a rotating fluid with Hall and ion-slip currents”, Wfirme- und Stofffibertragung, 1991, Vol 26, pp. 203205.

[15] N. Ghara, S. L. Maji,S. Das, R. N. Jana and S. K. Ghosh, "Effects of Hall Current and Ion-Slip on unsteady MHD Couette flow”, open journal of fluid dynamics, 2012, Vol 2, pp. 1 - 13.

[16] M. Kaviany,Principles of heat transfer in porous media., Springer Verlag, NewYork., 1991.

[17] H. Schlichting and K. Gersten, Boundary layer theory, Springer, 2006

[18] R.E. Bellman and R.E. Kalaba, Quasilinearization and Boundary-value Problems, Elsevier publishing Co. Inc., New York., 1965.

\section{Creative Commons Attribution License 4.0 (Attribution 4.0 International, CC BY 4.0)}

This article is published under the terms of the Creative Commons Attribution License 4.0

https://creativecommons.org/licenses/by/4.0/deed.en_US 